\title{
Three-dimensional analysis of flow and segregation control by slow rotation for Bridgman crystal growth in microgravity
}

\author{
C.W. Lan*, C.Y. Tu \\ Department of Chemical Engineering, National Taiwan University, Taipei 10617, Taiwan, ROC
}

\begin{abstract}
Although the buoyancy convection in the melt during crystal growth is greatly reduced in microgravity, the residual gravity, or the so-called $g$-jitter effect, can lead to three-dimensional (3D) unsteady flow and severe radial dopant segregation. Using a centrifuge to rotate the system about the growth axis could be an effective way to suppress the 3D flow and improve dopant uniformity. Through fully time-dependent 3D simulation of Bridgman growth of galliumdoped germanium crystals, we investigate the feasibility of using a centrifuge at low rotation rate to improve the dopant uniformity in the grown crystal. In this numerical model, in addition to the heat flow and the moving interface, both radial and axial segregations are also computed simultaneously for a growth period. The effect of slightly eccentric rotation is also considered and they can be an issue in practical implementation. (C) 2002 Elsevier Science B.V. All rights reserved.
\end{abstract}

PACS: 44.25. +f; 47.27.Te; 81.10.Fq; 02.60.c6; 02.70Fj

Keywords: A1. Computer simulation; A1. Convection; A1. Segregation; A2. Bridgman technique; A2. Microgravity conditions

\section{Introduction}

The control of heat flow, dopant segregation, and the growth interface is an important task in bulk crystal growth [1,2]. Using external forces, such as magnetic fields, to control crystal growth has been widely adopted [3-7]. Especially, if the buoyancy flow can be suppressed completely, the diffusion-controlled limit can be achieved. Accordingly, the axial segregation can be minimized if the growth distance is much longer than the diffusion length. With a careful control of interface shape to be flat, radial segregation can be further minimized

\footnotetext{
*Corresponding author. Tel./fax: +886-2-2363-3917.

E-mail address: lan@ruby.che.ntu.edu.tw (C.W. Lan).
}

as well. Microgravity is believed to an ideal environment to achieve this goal. However, experiments, as discussed in Ref. [8], have shown that the residual gravity or the so-called $g$-jitter effect, due to orbiting or the maneuver of astronauts, causes unsteady asymmetric flow and thus nonuniformities in the grown crystals. Therefore, even in microgravity, further flow suppression or control is still necessary. The use of magnetic fields should be helpful [9], but this requires the melt to be electrically conductive. Recently, a novel approach using steady ampoule rotation for flow and segregation control was proposed by Lan [10-11]. With a high enough rotation speed, he found that the diffusioncontrolled limit could be achieved for the vertical 
Bridgman (VB) configuration in normal gravity. Furthermore, through an extensive study [12], using a centrifuge to rotate the whole growth system about the growth axis was found to be the most effective way for flow and segregation control. In normal gravity, the rotation speed required for this application is high being in the order of $100 \mathrm{RPM}$. Therefore, if the idea is adopted for microgravity, it is believed that slow rotation $(<20$ RPM) should be enough for the same purpose. As will be shown shortly, the unsteady effect can be suppressed as well. Furthermore, since the rotation is about the growth axis, the centrifuge can be very compact and simple for microgravity experiments.

In this paper, we will present $3 \mathrm{D}$ simulation of heat flow and segregation in microgravity Bridgman growth of gallium-doped germanium with an arbitrary residual gravity. The effect of slow rotation is then considered to investigate its feasibility of flow and segregation control. In the next section, the numerical model is briefly described. Section 3 is devoted to results and discussion, followed by comments and conclusions in Section 4.

\section{Mathematical model and solution}

A Cartesian coordinate $(x, y, z)$ with the origin at the sample bottom is chosen; the $x$-axis is at the centerline of the sample. Based on the Boussinesq approximation, the governing equations in dimensionless form for the transport processes in the melt during crystal growth can be described by the conservation laws for the mass, momentum (in a rotating frame with a constant angular speed $\Omega$, i.e., $\boldsymbol{\Omega}=\boldsymbol{\Omega} \boldsymbol{e}_{x}$ here), energy, and dopant as follows:

$$
\nabla \cdot \boldsymbol{v}=0,
$$

$\frac{\partial \boldsymbol{v}}{\partial \tau}+\boldsymbol{v} \cdot \nabla \boldsymbol{v}=-\nabla P+\operatorname{Pr} \nabla^{2} \boldsymbol{v}+\boldsymbol{F}$,

$\frac{\partial T}{\partial \tau}+\boldsymbol{v} \cdot \nabla T=\nabla^{2} T$,

$\frac{\partial C}{\partial \tau}+\boldsymbol{v} \cdot \nabla C=\frac{P r}{S c} \nabla^{2} C$, where the body force term [12] can be written as follows:

$$
\begin{aligned}
\boldsymbol{F}= & -\operatorname{Pr} R a_{\mathrm{T}}\left(T-T_{\mathrm{m}}\right)\left[\boldsymbol{e}_{\mathrm{G}}+F r \boldsymbol{e}_{\mathrm{R}}\right] \\
& -\operatorname{Pr} T a^{1 / 2} \boldsymbol{e}_{\Omega} \times \boldsymbol{v} .
\end{aligned}
$$

In the above equations, $\boldsymbol{v}, \tau, P, T$, and $C$ are the dimensionless velocity, time, pressure, temperature and dopant concentration, respectively; $T_{\mathrm{m}}$ is the dimensionless melting temperature. Also, $\boldsymbol{e}_{\mathrm{G}}$ and $\boldsymbol{e}_{\mathrm{R}}$ are the unit vectors in the gravitational and centrifugal directions, respectively. In mircrogravity, $\boldsymbol{e}_{\mathrm{G}}=-\cos (\gamma) \boldsymbol{e}_{x}-\sin (\gamma) \boldsymbol{e}_{y}$ is adopted here (any form of $e_{\mathrm{G}}$ can be used) and $\gamma=2 \pi f \tau$ for simulating the $g$-jitter, where $f$ is the dimensionless jitter frequency. For steady-state calculations, $\gamma$ is set to be constant. The centrifugal acceleration can be represented as follows:

$\boldsymbol{e}_{\mathrm{R}}=\boldsymbol{\Omega} \times \boldsymbol{\Omega} \times(\boldsymbol{r}+\boldsymbol{R})$,

where $\boldsymbol{r}$ is the position vector $\left(\boldsymbol{r}=x \boldsymbol{e}_{x}+y \boldsymbol{e}_{y}+z \boldsymbol{e}_{z}\right)$ and $\boldsymbol{R}=R_{\mathrm{arm}} \boldsymbol{e}_{y}$. For the rotation about the growth axis, $R_{\mathrm{arm}}=0$. The associated dimensionless Rayleigh $\left(R a_{\mathrm{T}}\right)$, Taylor $(\mathrm{Ta})$, and Froude $(F r)$ numbers are defined as follows:

$$
\begin{aligned}
& R a_{\mathrm{T}}=\frac{\beta_{\mathrm{T}} g_{0} \Delta T R_{\mathrm{c}}^{3}}{v_{\mathrm{m}} \alpha_{\mathrm{m}}}, \quad T a=\frac{4 \Omega^{2} R_{\mathrm{c}}^{4}}{v_{\mathrm{m}}^{2}}, \\
& F r=\frac{\Omega^{2} R_{\mathrm{c}}}{g_{0}},
\end{aligned}
$$

where $R_{\mathrm{c}}$ is the crystal radius, $\Delta T$ the temperature difference between the hot and cold zones, $\mu_{\mathrm{m}}$ the melt viscosity and $g_{0}$ the reference gravitational acceleration; $g_{0}=10^{-5}-10^{-4}$ gal in this study. In addition, $\operatorname{Pr}=v_{\mathrm{m}} / \alpha_{\mathrm{m}}$ is the Prandtl number and $S c=v_{\mathrm{m}} / D$ the Schmidt number, where $v_{m}$ is the kinematic viscosity and $\alpha_{\mathrm{m}}$ and $D$ are the thermal and dopant diffusivities, respectively. For most of dopants in the melt, $S c \gg 1$. Therefore, the dopant field, and thus segregation, are dominated by the convection.

Apparently, from the body force term, if Fr $\boldsymbol{e}_{\mathrm{R}} \gg \boldsymbol{e}_{\mathrm{G}}$ (or $F r \gg 1$ ), then the $g$-jitter becomes less important, and the flow and segregation are controlled by rotation. However, the rotational acceleration $\left(r \Omega^{2}\right)$ can also generate the buoyancy flow, but it is driven by the axial thermal gradients. In addition, the last term in $\boldsymbol{F}$ is the Coriolis force 
and it always suppresses the flow. Detailed discussion of the interplay of the centrifugal acceleration and the Coriolis force can be found elsewhere [12]. With suitable boundary conditions, the above governing equations can be solved numerically by an efficient finite volume method with multigrid acceleration [13].

\section{Results and discussion}

For comparison purposes, we again take the growth of gallium-doped germanium $(\mathrm{GaGe})$ in the Grenoble furnace (with a linear heating profile) as an example. This problem, without rotation, was also investigated by Adornato and Brown [14]. Extensive benchmark numerical comparisons were also performed [15]. Therefore, it serves as a good candidate for the theoretical study here. The physical properties and some input parameters $\left(\Delta T=350^{\circ} \mathrm{C}\right)$ can be found elsewhere [15]. For pseudo steady-state calculations, where the growth speed is set to be the same the ampoule pulling speed $\left(U_{\mathrm{amp}}=-4 \times 10^{-4} \mathrm{~cm} / \mathrm{s}\right)$, the typical flow and dopant fields for $R a_{\mathrm{T}}=3.644$ (or $g_{0}=4.02 \times 10^{-5}$ gal) at two different gravity orientations $\left(\gamma=0\right.$ and $\left.90^{\circ}\right)$ are shown in Figs. 1a and c; $f=0 \mathrm{~Hz}$. As shown, if the gravity is pointing downward, no clear convection is induced except the velocity due to the ampoule translation. Accordingly, the dopant transport back to the bulk melt $(K=0.087)$ is diffusion limited. When the gravity is applied to the left (equivalent to the horizontal Bridgman configuration) for $\gamma=90^{\circ}$, as shown in Fig. 1c, a severe asymmetric flow and dopant nonuniformity are induced. Interestingly, with 10 RPM rotation $(F r=13.92 \gg 1)$, for both cases, the radial segregation $\left(C_{\mathrm{Cmax}}-C_{\mathrm{Cmin}}\right)$ is reduced. For $\gamma=0^{\circ}$, there is an upward flow near the wall, and this is due to the centrifugal acceleration $\left(r \Omega^{2}\right.$ is in the radial direction perpendicular to the axial thermal gradient). This flow also pushes the dopant near the interface outwards slightly. As a result, the original radial segregation due to the concave interface (Fig. 1a) is reduced. For $\gamma=90^{\circ}$ in Fig. 1d, beside the reduction of the radial segregation, the flow and dopant fields become much more axisymmetric. The dopant concentration in the grown crystal becomes more symmetric as well. Moreover, as shown by the dopant field in the $x-y$ plane near the interface, it is closer to the diffusion-limited one. If we take the dopant distribution at the interface along the direction from the maximum to minimum concentrations, as shown in Fig. 2 for $\gamma=90^{\circ}$, the improvement of radial segregation by rotation becomes clearer. The results for 5RPM and $20 \mathrm{RPM}$ are also included for comparison. As shown, with a certain rotation rate, radial segregation can be reduced. In this case, 10 RPM seems to be a good choice. Rotation at 20 RPM, though improves the symmetry, starts to increase segregation due to the stronger buoyancy flow induced by centrifugal acceleration.

Beside the pseudo-steady-state calculations, fully time-dependent calculations are also carried out. As such, in addition to radial segregation, axial segregation can also be evaluated. For comparison purposes, the growth starts at the middle of the sample (at $x=3.5 \mathrm{~cm}$ ). The growth is ended at $4500 \mathrm{~s}$ for comparison (half of the melt is solidified), which corresponds to the growth distance being about $1.8 \mathrm{~cm}$; the heater moving speed $U_{\mathrm{h}}=4 \times 10^{-4} \mathrm{~cm} / \mathrm{s}$. The axial segregation (averaged concentration) as well as the maximum and minimum concentrations are shown in Fig. 3 for the case with and without rotation (20 RPM); the jitter frequency is set to be $10^{-3} \mathrm{~Hz}$ and gravity level $10^{-4}$ gal (larger than that in Fig. 1). As shown, for the case without rotation, significant concentration oscillation is found and the period is about $2 \mathrm{~mm}$ (or $500 \mathrm{~s}$ ); the gravity orientation takes $500 \mathrm{~s}$ to change from $0^{\circ}$ to $180^{\circ}$. As illustrated in Fig. 1c, the strongest convection occurs at $\gamma=90^{\circ}$, where the gravity is perpendicular to the thermal gradients. The largest radial segregation $\left(\Delta C_{\mathrm{C}}\right)$ is about $50 \% ; \Delta C_{\mathrm{C}}=\left(C_{\mathrm{Cmax}}-\right.$ $\left.C_{\mathrm{Cmin}}\right) / C_{0}$. With $20 \mathrm{RPM}$ rotation $(F r=22.36)$, it is clear that the oscillation amplitude is greatly reduced. Furthermore, the result is closer to the diffusion-limited one, which is also included for comparison. For smaller rotation speed and gravity level, the axial segregation can be further closer to the diffusion limited one. On the other hand, the $g$-jitter enhances convection and thus dopant mixing. 


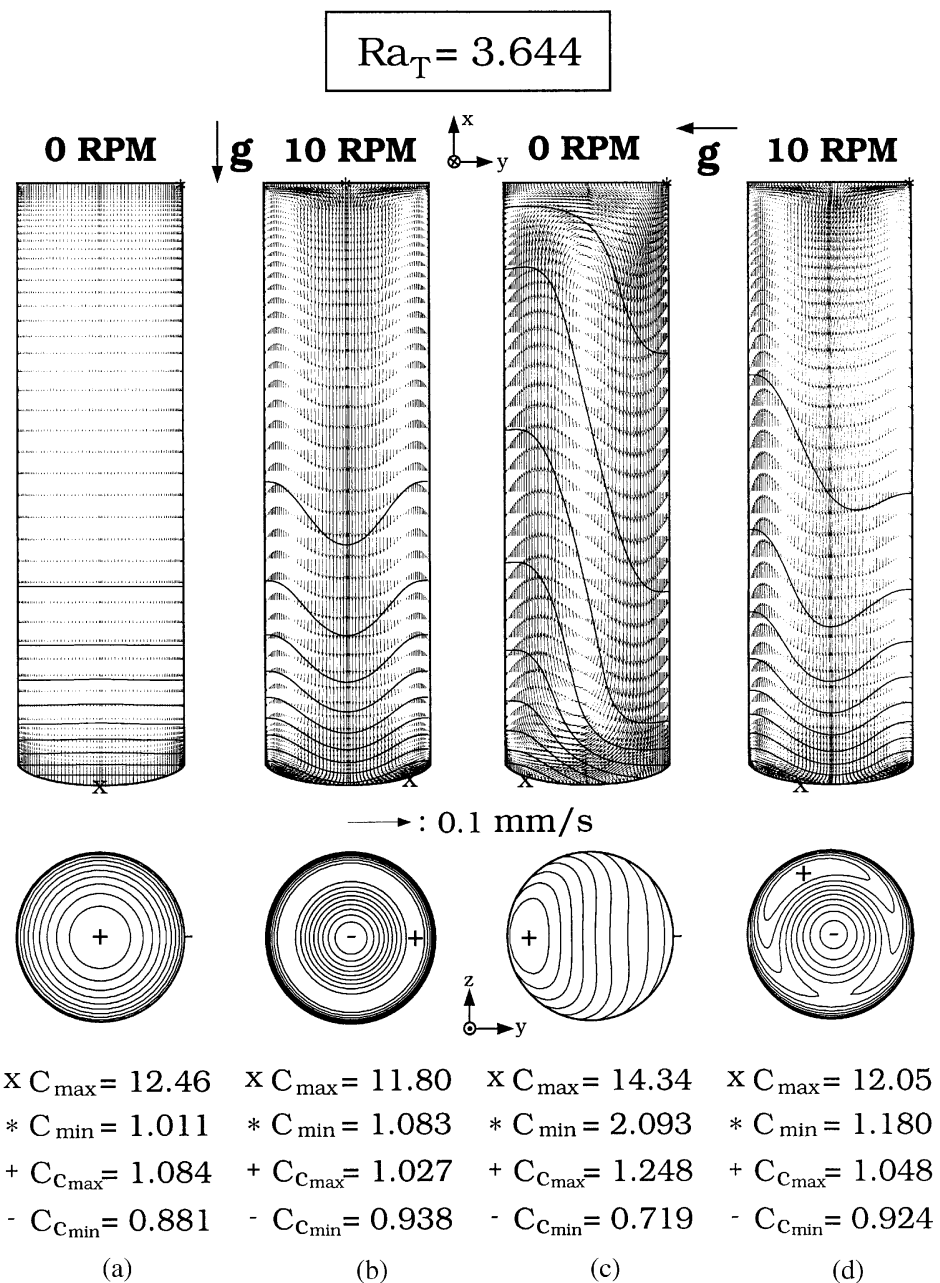

Fig. 1. Effect of 10 RPM rotation on flow and dopant fields: (a) $\gamma=0^{\circ}, 0 \mathrm{RPM}(F r=0)$; (b) $\gamma=0^{\circ}, 10 \mathrm{RPM}(F r=13.92)$; (c) $\gamma=90^{\circ}$, 0 RPM $(F r=0)$ and (d) $\gamma=90^{\circ}, 10$ RPM $(F r=13.92) ; U_{\text {amp }}=-4 \times 10^{-4} \mathrm{~cm} / \mathrm{s}$ and the lower figures are the dopant distribution $\left(C_{\mathrm{C}}=K C\right)$ in the grown crystal.

The final issue needs to be concerned in practice is the eccentric rotation because a perfect arrangement may not be possible. If the rotation axis is slightly off, the centrifugal acceleration becomes asymmetric due to the nonzero $R_{\text {arm }}$. We have performed two additional calculations for $R_{\text {arm }}=$ 0.5 and $1 \mathrm{~mm}$. The result becomes more asymmetric with the increasing eccentricity. Nevertheless, if $R_{\text {arm }}$ is $<0.5 \mathrm{~mm}$ (about $10 \%$ of the crystal radius), the eccentric effect may be neglected. Interestingly, the result of $R_{\text {arm }}=0.5 \mathrm{~mm}$ shows the least radial segregation among the examples.

\section{Conclusions}

In this paper, we perform $3 \mathrm{D}$ calculations to investigate the feasibility of using slow rotation to suppress the $g$-jitter effect on the Bridgman crystal growth (or gradient-freeze growth). As illustrated, in addition to asymmetry due to the arbitrary gravity orientation, the unsteady nature of the $g$ jitter is greatly suppressed by rotation. Much more axisymmetric flow and dopant fields can be obtained. Furthermore, for the case with a concave interface, the convection induced by the centrifugal acceleration also pushes the dopant 


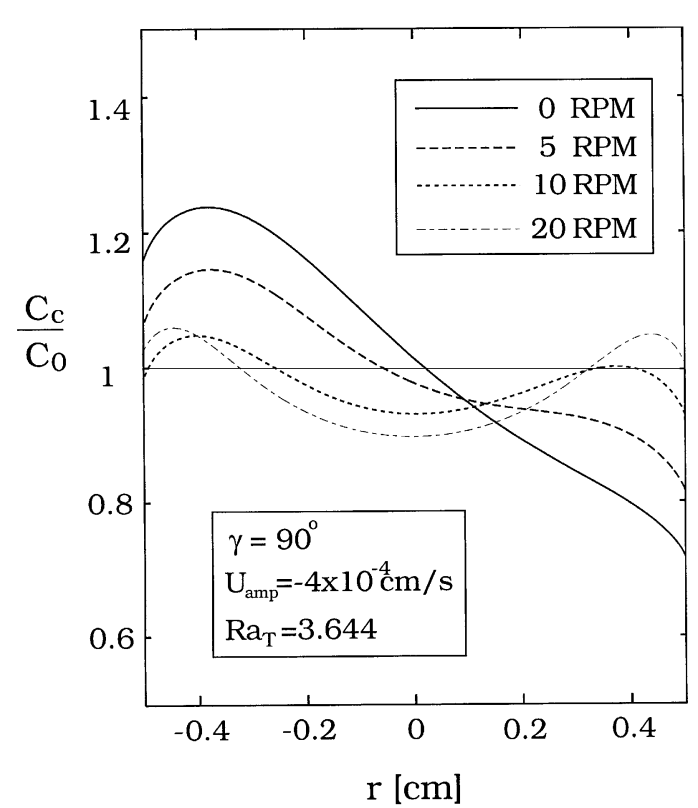

Fig. 2. Effect of rotation speed for the horizontal gravity $\left(\gamma=90^{\circ}\right)$ on the radial dopant distribution (along the maximum and minimum directions); the results for 0 and 10 RPM are taken from Figs. 1c and d.

outward, which minimizes the radial segregation due to the interface concavity. With an optimum rotation speed, a uniform dopant distribution may be obtained. More importantly, besides the radial segregation, the axial segregation with rotation is closer to the diffusion-controlled limit. As a result, if the growth distance is long enough being several diffusion distances, axial segregation can be minimized. Finally, eccentric rotation induces asymmetric flow. However, the calculations show that if the rotation axis does not deviate much from the growth axis, one still gains the advantages of slow rotation.

\section{References}

[1] R.A. Brown, A.I.Ch.E. J. 34 (1989) 881.

[2] G. Müller, A.in Ostrogorsky, in: D.T.J. Hurle (Ed.), Handbook of Crystal Growth 2b: Growth Mechanisms and Dynamics, North-Holland, Amsterdam, 1994.

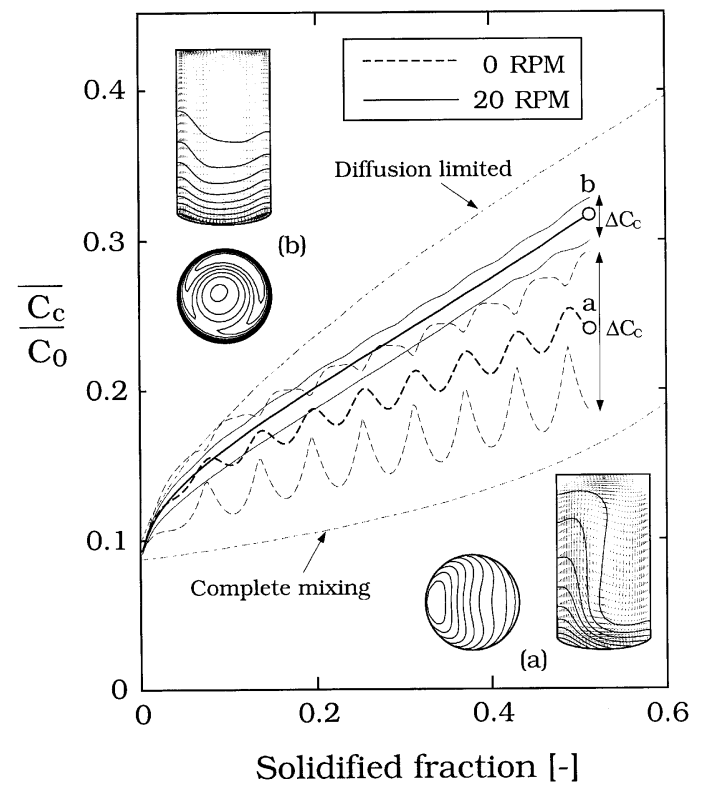

Fig. 3. Effects of rotation on axial (averaged value) and radial segregation under $g$-jitter; $f=10^{-3} \mathrm{~Hz}, R a_{\mathrm{T}}=9.073$, and $\Delta C_{\mathrm{C}}=\left(C_{\mathrm{Cmax}}-C_{\mathrm{Cmin}}\right) / C_{0}$. The flow and dopant fields at the end of growth are also shown.

[3] H.P. Utech, M.C. Flemming, J. Appl. Phys. 37 (1966) 2021.

[4] K.M. Kim, J. Electrochem. Soc. 132 (1982) 427.

[5] D.H. Kim, P.M. Adornato, R.A. Brown, J. Crystal Growth 89 (1988) 339.

[6] H.J. Scheel, J. Crystal Growth 13/14 (1971) 560.

[7] C.W. Lan, Int. J. Heat, Mass Transfer 43 (2000) 1987.

[8] D.T.J. Hurle, G. Muller, R. Nitsche, in: H.U. Walter (Ed.), Fluid Sciences and Materials Science in Space, A European Perspective, Springer, Berlin, 1987, p. 315.

[9] T. Duffar, J. Ph. Nabot, C. Barat, J.J. Favier, G. Cambon, Microgravity Sci. Technol. VI/2 (1993) 74.

[10] C.W. Lan, J. Crystal Growth 197 (1999) 983.

[11] C.W. Lan, J. Crystal Growth 229 (2001) 595.

[12] C.W. Lan, C.Y. Tu, J. Crystal Growth 226 (2001) 406.

[13] C.W. Lan, M.C. Liang, J. Comput. Phys. 152 (1999) 55.

[14] P.M. Adornato, R.A. Brown, J. Crystal Growth 80 (1987) 155.

[15] C.W. Lan, F.C. Chen, Comput. Methods Appl. Mech. Eng. 131 (1996) 191. 